die neuzeitliche Politik zeigen, dass Politik und Theologie sich wechselseitig aufeinander beziehen. $\mathrm{Zu}$ suggestiv bleibt allerdings die Grundthese des Buches, dass eine Genealogie des ,politischen Dispositivs der heutigen Demokratien“ (12) vorgelegt werde. Denn so interessant die Verweise auf die Theoreme und historischen Argumentationen auch sind, so bieten sie doch an sich keinen Grund dafür, anzunehmen, dass die moderne Demokratie nicht auch ohne sie zu begreifen wäre. Und gerade der apokalyptische Ton der Darstellung, der schon die anderen Bände der Homo Sacer-Reihe geprägt hat und insinuiert, gegenwärtige Demokratien gingen ihrem Untergang entgegen, wenn sie ihre Genealogie nicht angemessen verständen, ist weiterhin äußerst störend.

\section{Andreas Niederberger}

\section{Bering, Dietz. Die Epoche der Intellek- tuellen 1898-2001, Geburt - Begriff - Grabmal. Berlin. Berlin University Press 2010. 756 Seiten. 49, $90 €$.}

Glaubt man den Edelfedern unserer Feuilletons, so ist es mit dem „Zeitalter der Intellektuellen “ zu Ende. Seitdem alle „Großen Erzählungen“ und damit das Zeitalter der Ideologien vergangen seien, habe auch die einstige Figur des bürgerlichen, gar des proletarischen Intellektuellen das Zeitliche gesegnet.

Begründet wird diese freudige Todesbotschaft vor allem mit dem Niedergang sämtlicher Sozialutopien, wie sie noch im 19. und 20. Jahrhundert wirksam waren: allen voran der Sozialismus mitsamt dem „sozialdemokratischen Zeitalter", erst recht der Kommunismus und Bolschewismus des vorigen Jahrhunderts. Aus alldem wird gefolgert, dass heute statt der Intellektuellen nur mehr Experten und nüchterner Pragmatismus statt utopischer Projektionen gefragt sind. Angesagt sei gegenwärtig ein entschiedener Antiutopismus, der sich als „postideologisch“ und von Grund auf wirklichkeitsorientiert versteht, so wie ihn etwa Joachim Fest im Jahre 1991 mit seinem Pamphlet „Der zerstörte Traum. Vom Ende des utopischen Zeitalters" propagiert hat. Wenn es ein Merkmal gibt, dass man von Seiten unzähliger konservativer Nachrufe den Intellektuellen bis heute zuschreibt, so ist es ihr angeblicher Realitätsverlust, an dem sie letztlich scheitern müssten.

Der Zusammenbruch des Sowjetreiches hatte so denn auch einen doppelten Effekt: Er galt weithin als epochaler Beweis, dass alle gesellschaftspolitischen Ideologien und Utopien der Neuzeit und damit die Moderne selbst von Anfang an zum Scheitern verurteilt gewesen seien. Träger dieser Projektionen waren seit je die Intellektuellen, die damit ihre einstigen Funktionen eingebüßt hätten. Vor dem Hintergrund dieses gleichermaßen populären wie schematischen Dualismus: hier Realität - da Intellektuelle, erscheint das hier anzuzeigende Buch von Dietz Bering zur rechten Zeit, um die fällige Klärung und Differenzierung auf einem in der Tat recht unübersichtlichen Gelände einzuleiten. Der Autor hatte mit seiner bereits 1978 erschienenen Schrift „Die Intellektuellen. Geschichte eines Schimpfwortes" (Stuttgart) eine beachtliche Vorarbeit geleistet, die er nun systematisiert und in einem mehr als 750-seitigen Werk auf eine auch empirisch breitere Grundlage stellt. Dass dies nicht - wie meist üblich - in nur essayistischer Form geschieht, zeigt bereits ein Anhang, worin Bering, 
gleichsam zur quellenkritischen Selbstprüfung, ein „Hieb- und Stichwort-Register" der Intellektuellenfeinde und ein komplementäres „Gegen-Schlagwortregister" der Intellektuellen selbst bietet. Schon mit diesem nützlichen Instrumentarium, das man bisher in den Beiträgen zum Thema vermisste, wird $B e$ rings Werk zu einer Art Kompendium und Vademecum auch der Intellektuellensoziologie.

Seine Darstellung beginnt der Autor mit der „Geburt“ des modernen Intellektuellen in der Dreyfus-Affäre (1898). Sodann schildert er eingehend den Weg hin zur Weimarer Republik und zum Nationalsozialismus. Das Kapitel über die Periode nach dem Zweiten Weltkrieg führt über die Gruppe 47 zu den Achtundsechzigern, schließlich zur Frage des angeblichen Schweigens der Intellektuellen zur Zeit des Mauerfalls (445 ff.). Für viele deutsche Leser dürfte besonders der letzte Teil, der bis zur Jahrhundertwende reicht, interessant sein, wo unter anderem Autoren der französischen Intellektuellendiskurse wie Foucault oder Lyotard thematisiert werden.

Vom deutschen Historiker Jörn Rüsen wird erzählt, dass er, gefragt, weshalb gerade heute wieder über Utopie gesprochen werden sollte, antwortete: „Weil sie totgesagt wurde und doch zum Leben der Kultur gehört“. Mit dieser Devise entlässt auch Bering seine Leser: Noch nie seien Intellektuelle nötiger gewesen als in einer Zeit wie der unseren, in der Globalisierung, Ökokrisen, Finanz- und Wirtschaftskatastrophen einander ablösen. Darin trifft der Autor sich mit Carlo Schmid, dem einstigen Groß-Intellektuellen in der deutschen Sozialdemokratie, der in den fünfziger und sechziger Jahren nicht müde wurde, die historischen
Leistungen von Intellektuellen zu würdigen und in seinem Buch „Vorurteile gegen Intellektuelle“ von 1970 schrieb: „Der Intellektuelle ist einer, der nicht bereit ist, die Welt, die er antrifft, einfach schon darum anzuerkennen und für bestätigt zu halten, weil sie eben da ist. Er wird also dem bloßen, Gewordensein' für sich allein keinerlei rechtfertigenden Charakter zuerkennen. Er wird dem, was ist, sofern es Menschenwerk ist, immer mit dem Richtscheit der Vernunft und der Moral begegnen und sich nicht damit abfinden, dass etwas schon darum vernünftig sein soll, weil es wirklich ist und gemeinhin akzeptiert wird“ (351 f.). Carlo Schmid hielt die spezifische Funktion von Intellektuellen in der Demokratie für unentbehrlich, denn der Staat sei eine Ordnung, die ohne die lebendige Beteiligung gerade intellektueller Schichten gar nicht denkbar sei. Allerdings hatte Schmid noch einen vergleichsweise emphatischen Begriff vom Intellektuellen: Es sei ihm auferlegt, ,die Ansätze zu Ende zu denken, seine analytische Kraft muss also bis auf den Grund gehen. Die Aufgabe: sich dagegen zu wehren, dass der Mensch zu einem bloßen Objekt seelenloser Mechanismen reduziert wird [...] nur so könne der Staat aus der Versteinerung gelöst werden “ (338 f.).

Eines der interessantesten Ergebnisse der Arbeit Berings bezeichnet den grundlegenden Unterschied zwischen der Situation der Intellektuellen in Frankreich und Deutschland. Während es nämlich den französischen Intellektuellen schon in der Dreyfus-Affäre gelungen war, sich das ursprünglich von ihren Gegnern blindwütig benutzte Verdikt „Intellektueller“ als Selbstund Ehrenbezeichnung anzueignen hierin nicht unähnlich den zeitgenössi- 
schen „Expressionisten“ -, haben es die deutschen Intellektuellen in ihrer Mehrheit eigentlich nie vermocht, sich durch semantische Umwertung aus dem sozialen Ghetto pejorativer Ausgrenzung zu befreien. Ludwig Erhards "Pinscher" und „Schmeißfliegen “ und die Rede Konrad Adenauers von einem "Abgrund von Landesverrat" in der Spiegel-Affäre zeigen, weshalb deutsche Intellektuelle auch nach dem Zweiten Weltkrieg aus ihrer permanenten Defensivhaltung nicht wirklich herausfinden konnten.

Aufschlussreich ist Berings Beobachtung, dass die Rede vom „Grabmal der Intellektuellen “ (Lyotard) gegenwärtig konterkariert wird durch eine kaum nachlassende „Reizwirkung“ des Wortes selbst. Bereits aus dieser - offenbar nach wie vor unterschwelligen - Brisanz des Intellektuellenbegriffs ließe sich womöglich schließen, dass weniger das Ende als vielmehr eine Metamorphose dieses doch wohl überaus lernfähigen Menschentyps zu erwarten ist. Eher spricht manches dafür, dass das, wofür Intellektuelle seit je standen, angesichts neuer globaler Bedrohungen zu einer Sache aktiver Bevölkerungsminderheiten selbst werden könnte, so wie sich dies in jüngster Zeit an zahlreichen Rebellionen gerade besser situierter Bürger auch in unserer Republik ablesen lässt. Denn Intellektuelle waren stets jene, die über ihre spezifische Fachkompetenz hinaus zugleich den Zustand der Gesamtgesellschaft bedachten und deshalb - spätestens seit Emile Zola - oft nicht umhin konnten, sich in bestimmten Situationen für Menschenrechte, kollektive Bedürfnisse und gegen soziale und politische Defizite zu engagieren.

Die allerorten bis zum Überdruss propagierte „Alternativlosigkeit“ gegen- wärtiger politischer Entscheidungen, wie sie schon von Margret Thatcher einst (als „TINA-Prinzip“) öffentlichkeitswirksam verkündet worden war, bildet eines der zentralen Fermente heutiger Ideologiehaftigkeit, die dem gleichzeitig behaupteten Ende aller Ideologien Hohn spricht. Denn aus dieser Optik erscheinen die heutigen ökonomischen und politischen Prozesse schlechthin als Naturereignisse bzw. als „verhängtes Schicksal“, demgegenüber jeglicher Widerstand von vornherein müßig erscheine (Oswald Spengler lässt grüßen!).

Freilich weist Berings Werk auch einige Lücken auf, deren eine künftige Forschung sich annehmen müsste. Dies betrifft zum einen die fällige Erforschung der intimen, apokryphen Affinität von gängiger Intellektuellenfeindschaft und modernem Antisemitismus (dem übrigens Bering andernorts einige Untersuchungen gewidmet hat), eine Nähe, wie sie nicht allein im Wilhelminischen Deutschland um die Wende zum 20. Jahrhundert offenbar wurde (vgl. etwa Houston Stewart Chamberlain). Eine zweite Frage bedürfte einer noch genaueren Untersuchung: nämlich die Situation der Dissidenten in ehedem stalinistisch organisierten östlichen Ländern. Material hierzu könnten noch zahlreiche Zeitzeugen liefern. Ihre biografischen Erinnerungen harren nach wie vor einer systematischen Aufarbeitung.

Beide Anmerkungen schmälern jedoch die Leistung des Autors, seines Zeichens Sprachwissenschaftler, nicht. Die Beobachtung, dass hier ein unbefangener Forscher aus einer geisteswissenschaftlichen Disziplin sich eines soziologisch wie politisch nach wie vor brisanten Themas annimmt, sollte Anlass sein, die kreative Funktion 
sogenannter Außenseiter erneut zu reflektieren. Sie gerade könnten „Schwachstellen“ etablierter Disziplinen sichtbar machen.

\section{Kurt Lenk}

Condorcet, Marquis de. Freiheit, Revolution, Verfassung. Kleine politische Schriften. Hrsg. von Daniel Schulz. Berlin. Akademie Verlag 2010. 276 Seiten. 69,80€.

\section{„Der Akademiker Condorcet“ sei} „einstmals ein großer Mathematiker in den Augen der Literaten und ein groBer Literat in den Augen der Mathematiker" gewesen. Dieses Diktum Robespierres könnte eine Aversion gegen den mehrfach begabten Condorcet aussprechen. Mathematiker, Aufklärer und Philosoph, politischer Denker und politischer Praktiker war er. Und das auch noch in Verbindung. Das nach ihm benannte Condorcet-Paradoxon, seine Memoranden zur republikanischen und weltlichen Volksbildung von 1792, die maßgeblich von ihm verfasste "Girondine“ (der Verfassungsentwurf von 1792/93, der aufgrund der Errichtung der Jakobinerherrschaft nach dem 2.6.1793 nicht mehr zum Tragen kam), sein in der Verfolgungszeit geschriebener, als reine Apologie des Fortschritts und des instrumentellen Kalküls schlicht missverstandener geschichtsphilosophischer Entwurf auf Turgots Spuren, den die Thermidorianer 1795 posthum veröffentlichten: „Entwurf einer historischen Darstellung der Fortschritte des menschlichen Geistes“ (deutsch Köln 1963), sind den Lexika noch bekannt. Letzteres ist ein Irrtum, weil dieser dialektische „Esquisse " für die Ambivalenzen des Fortschritts überaus sensibel ist.
Aber sonst? Vielfach wurde und wird Condorcet von der Rezeption sogar der politischen Ideengeschichte eher in die zweite Reihe des politischen Denkens gestellt.

Die Sicht auf den politischen Denker Condercet ist auch für Nicht-Französischkundige nun dank der von Daniel Schulz edierten und eingeleiteten vorzüglichen Auswahl-Ausgabe etwas besser möglich, die Condorcet vornehmlich als "deliberativen“ Demokratietheoretiker zeigt (43), der „für das heutige Verständnis des Verfassungsstaats immer noch von Bedeutung" ist (9). Dies, indem er komplementär zur repräsentativen Staatskonzeption der Jakobiner - die allerdings die sansculottische Volksbewegung zeitweilig im Sinne einer Doppelstrategie dazu benutzten, um im Konvent ihre jeweiligen Gegner zu besiegen - durch eine „Kombination kleinräumiger Partizipation und großräumiger Repräsentation “ wirkungsvolle, dabei immer vermittelte und nicht etwa direktdemokratische institutionelle Passagewege bürgerschaftlicher Partizipation entwirft, ,[...] um dem Bürger als aktivem Teilnehmer des politischen Geschehens einen angemessenen Raum in der politischen Ordnung zu verschaffen ", der nicht nur ein fehlerfreundliches Vetorecht, sondern auch eine Gesetzesinitiative der Bürger beinhaltet. Er biete so eine Schnittstelle von republikanischen und liberalen Elementen, ohne sie gegeneinander auszuspielen, so Schulz $(43,37)$.

Sparsam, jedoch zureichend erläutert kommt der schmale Band daher. Zwar ist er nicht chronologisch, sondern unter Pertinenzgesichtspunkten gemäß seinem Untertitel gegliedert, bleibt aber dennoch übersichtlich. Damit wird die Entwicklung der politischen Konzep- 